\title{
A one-year survey of Neisseria gonorrhoeae isolated from patients attending an east London genitourinary medicine clinic: antibiotic susceptibility patterns and patients' characteristics
}

\author{
D A Lewis, C A Ison, D M Livermore, H Y Chen, A Y Hooi, A R Wisdom
}

\begin{abstract}
Objectives-To collect epidemiological data on gonococcal infection in an east London genitourinary medicine (GUM) clinic; to perform antibiotic susceptibility testing on Neisseria gonorrhoeae isolates and relate results to patient data; to assess the efficacy of current first-line antibiotic therapy for treating gonorrhoea.
\end{abstract}

Methods-Gonococcal isolates were collected from 113 patients attending the GUM clinic at Newham General Hospital over a one year period. Isolates (104) were tested for susceptibility to various antibiotics. Plasmid profiles were obtained for penicillinase producing gonococci (PPNG) and isolates exhibiting high-level tetracycline resistance (TRNG). Epidemiological information was collected from clinic attenders by routine note-taking.

Results-PPNG (16) accounted for $15 \%$ of isolates tested, only three being acquired outside the United Kingdom (U.K.). Plasmid typing showed three types of $\beta$-lactamase-encoding plasmids were represented $(2 \cdot 9 \mathrm{MDa}, 3 \cdot 2 \mathrm{MDa}$ and 4.4 MDa). Amongst the non-PPNG isolates, high-level chromosomal resistance to penicillin (CMRNG) was found in $3.5 \%$, intermediate resistance in $57.5 \%$ and full susceptibility in $39 \%$. One isolate showed decreased susceptibility to ciprofloxacin (MIC $=0.06 \mathrm{mg} / \mathrm{l}$ ). Three PPNG isolates also possessed a $25 \cdot 2 \mathrm{MDa}$ plasmid and expressed high-level tetracycline resistance encoded by tetM. All isolates were susceptible to cefixime, cefotaxime, azithromycin and spectinomycin. Most gonorrhoea $(90 \%)$ was seen in local residents. The male:female case ratio was 2:1 with homosexually-acquired gonorrhoea accounting for only $3 \cdot 5 \%$ of the total. Most patients $(96 \%)$ had acquired gonorrhoea in the U.K.. A past history of gonorrhoea was more frequent in male patients. Concurrent chlamydial infection was seen in $31 \%$ females and $16 \%$ males.

Conclusions-The high PPNG rate supports a previous decision to change firstline therapy from amoxycillin with probenecid to ciprofloxacin. There was no evidence of clinical treatment failure with ciprofloxacin. Cefixime, cefotaxime, azithromycin and spectinomycin all appear to be suitable alternative thera- pies. Acquisition of gonorrhoea abroad was associated with isolates exhibiting penicillin resistance but such isolates were also obtained from patients infected locally and without a history of foreign travel.

(Genitourin Med 1995;71:13-17)

Keywords: Gonorrhoea, Resistance, Epidemiology

Introduction

Penicillin has been used as first-line therapy for gonorrhoea for over forty years. However, the increase in both chromosomally- and plasmid-mediated resistance to this drug led to a change in the World Health Organisation (WHO) treatment recommendations in 1989. ${ }^{1}$ These WHO guidelines now recommend one of the following single-dose regimens as first-line therapy: ciprofloxacin 500 mg orally, ceftriaxone $250 \mathrm{mg}$ intramuscularly (i.m.) or spectinomycin $2 \mathrm{~g}$ i.m.. Penicillin is only recommended in areas where resistance is rare. Tetracycline is widely used in many parts of the world, especially where the WHO recommended agents are not available or too expensive. However, high-level plasmid-mediated resistance to tetracycline in Neisseria gonorrhoeae, first described in 1985 in the USA, ${ }^{2}$ is now spreading worldwide, undermining this treatment.

The genitourinary medicine (GUM) clinic at Newham General Hospital serves a population of diverse ethnic background in the east end of London. Ciprofloxacin as a $250 \mathrm{mg}$ single oral dose has been first-line therapy for the treatment of gonorrhoea in men and non-pregnant women since 1987. Pregnant women are usually treated with two $3 \mathrm{~g}$ doses of amoxycillin, given 24 hours apart. Spectinomycin is occasionally used as secondline therapy.

The aim of this study was to determine the susceptibility of all $N$ gonorrhoeae isolated in this clinic during one year to a range of antibiotics and to relate the findings to demographic data collected from the infected patients. The efficacy of current treatment regimens for antibiotic-resistant gonorrhoea was assessed.

Materials and methods

Isolation of Neisseria gonorrhoeae

Specimens were taken using a disposable loop (urethral) or cotton tipped swab (cervical, rec- 
tal and pharyngeal) and inoculated directly onto "GC medium" containing Columbia agar base (Unipath, Basingstoke, Hants. UK) with $5 \%$ defibrinated lysed horse blood (Unipath) and antibiotics as follows: vancomycin, $3 \mathrm{mg} / 1$; colistin, $7.5 \mathrm{mg} / 1$; nystatin, $12,500 \mathrm{U} / 1$ and trimethoprim, $5 \mathrm{mg} / \mathrm{l})$. Plates were incubated immediately in a humidified incubator at $37^{\circ} \mathrm{C}$ in an atmosphere of $5 \%$ $\mathrm{CO}_{2}$ for up to 48 hours. Typical colonies of Gram negative cocci that were oxidase positive were identified as $N$ gonorrhoeae following carbohydrate utilisation studies. Isolates from 113 patients were subcultured on to "GC medium" without added antibiotics and stored at $-70^{\circ} \mathrm{C}$ in microbank vials (Pro-Lab Diagnostics, Bromborough, Wirral, UK). Subculture from $-70^{\circ} \mathrm{C}$ was onto freshlymade GC agar (Unipath) with 5\% defibrinated horse blood, pre-lysed with $0 \cdot 1 \%$ saponin. All presumptive gonococcal isolates were reidentified with Gram staining, oxidase testing and carbohydrate utilisation. Nine isolates failed to grow after subculture from the vials. Isolates were re-subcultured twice more before determining antibiotic susceptibility or plasmid profiles.

\section{Screening for other STDs}

Urethral and cervical specimens were taken for chlamydial culture. The presence of Chlamydia trachomatis within McCoy cell monolayers (ICN Flow, High Wycombe, Bucks, UK) was demonstrated by iodine staining for inclusion bodies after appropriate incubation. Females had high vaginal swabs taken to demonstrate Trichomonas vaginalis on wet film. Patients were screened for treponemal disease using a combination of the non-specific Venereal Diseases Research Laboratory test (VDRL; H. D. Supplies, UK) and a specific Treponema pallidum haemagglutination assay (Fujirebio, Mast Group, UK). Presumptive positive sera were further tested with a specific fluorescent treponemal antibody test (FTA; treponemal antigen from Wellcome, UK and fluorescein-conjugated sheep anti-human globulin from Binding Site Ltd., UK). Patients screened within the last year were not re-screened unless treponemal disease was suggested clinically.

Minimum inhibitory concentrations (MICs)

MICs were determined by an agar dilution technique on GC agar (Unipath) with 5\% lysed horse blood and added antibiotics at appropriate concentrations. Antibiotics tested included benzylpenicillin, $0.001-32 \mathrm{mg} / \mathrm{l}$; cefotaxime (Roussel), 0.001-1 mg/l; cefixime (Lederle), $\quad 0.001-1 \mathrm{mg} / \mathrm{l}$; spectinomycin (Upjohn), 4-256 mg/l; ciprofloxacin (Bayer), $0.001-1 \mathrm{mg} / \mathrm{l}$; tetracycline, $0.06-16 \mathrm{mg} / \mathrm{l}$ and azithromycin (Pfizer), 0.004- $2 \mathrm{mg} / \mathrm{l}$. In control plates, sterile water replaced the antibiotic solutions. Inocula were prepared by removing colonies from an overnight plate cultures and preparing turbid suspensions in nutrient broth (Unipath) to equate visually with the turbidity of a McFarland 1.0 standard. A multipoint inoculator (Mast Diagnostics, Bootle,
Merseyside, UK) was used to inoculate the plates with $10^{5}$ c.f.u./spot. WHO control gonococcal strains A-E with known susceptibility or resistance to penicillin, spectinomycin and ciprofloxacin were used as controls in each MIC run. Plates were incubated at $37^{\circ} \mathrm{C}$ in $5 \% \mathrm{CO}_{2}$ and read 24 hours later. The MIC was defined as the lowest concentration of antibiotic at which growth of the gonococci was inhibited. All MIC determinations were performed twice on separate occasions.

\section{$\beta$-lactamase testing}

All isolates were tested for $\beta$-lactamase with a $0.1 \mathrm{mM}$ nitrocefin solution.

\section{Plasmid profiles}

All $\beta$-lactamase-positive isolates and those exhibiting high-level tetracycline resistance (MIC $\geqslant 16 \mathrm{mg} / \mathrm{l}$, presumed TRNG) had plasmids extracted using the method of Birnboim and Doly. ${ }^{3}$ The cultures used were grown overnight initially on GC agar (Difco, West Molesey, Surrey, UK) plus 1\% Iso VitaleX and half the growth was added to 1 $\mathrm{ml}$ of sterile saline before commencing the plasmid extraction. Control strains of $N$ gonorrhoeae containing $2.9 \mathrm{MDa}, 3 \cdot 2 \mathrm{MDa}$ or 4.4 $\mathrm{MDa} \beta$-lactamase plasmids were prepared similarly as were control strains containing either the $24.5 \mathrm{MDa}$ conjugative plasmid or the $25 \cdot 2 \mathrm{MDa}$ tetracycline resistance plasmid. All plasmid extracts were electrophoresed at $120 \mathrm{~V}$ in agarose gel ( $1 \%$ for PPNG, $0.8 \%$ for presumed TRNG). The gels were stained with ethidium bromide $(1 \mathrm{mg} / \mathrm{l})$, viewed under UV light and polaroid photography performed.

\section{Polymerase chain reaction (PCR) for tet $M$}

PCR was performed on dilutions of whole cells harvested from an overnight growth on GC agar using a method described previously. ${ }^{4}$ All isolates expressing high-level tetracycline resistance (MIC $\geqslant 16 \mathrm{mg} / \mathrm{l}$ ) were tested. Appropriate positive (a known TRNG strain provided by Dr. Joan Knapp, Center for Diseases Control and Prevention, Atlanta, USA) and negative controls were used in the PCR test. Thermal cycling was performed in the Hybaid Omnigene (Hybaid Ltd., Teddington, UK). Two primers based on the sequence of the Ureaplasma urealyticum tetM were used to amplify the gonococcal tet $M$ gene sequence. PCR products were analysed by electrophoresis of a $10 \mu \mathrm{l}$ aliquot of reaction mixture on $1 \%$ agarose gel. Molecular weight markers (1 kilobase ladder, Life Technologies, Paisley, Scotland) were used on each gel.

\section{Patient data}

The notes of all patients from whom gonococci were isolated were reviewed to allow collection of relevant epidemiological and clinical data. For each patient these included age, postcode of residence, ethnicity, sex, geographical location of infection with gonorrhoea, history of foreign travel over the previous six months, history of previous gon- 


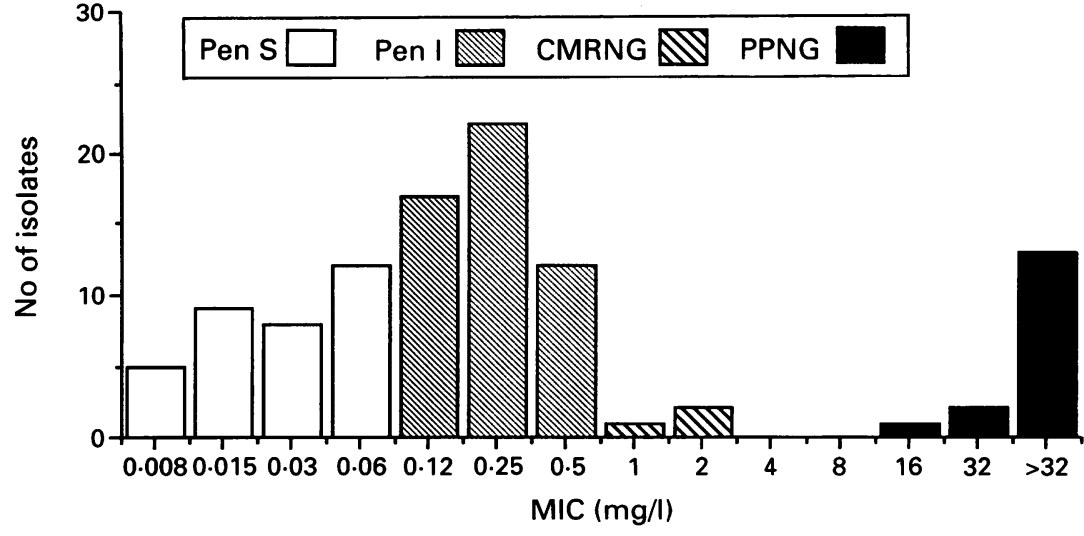

Penicillin MIC distribution for gonococci isolated from NGH patients ( $n=104)$.

Susceptibility to penicillin is divided into four internationally recognised groups by MIC data and $\beta$-lactamase testing: full susceptibility (Pen $S, M I C \leqslant 0.06 \mathrm{mg} / \mathrm{l}$ ), intermediate susceptibility (Pen I, MIC 0.12-0.5 mg/l), chromosomal resistance (CMRNG, MIC $\geqslant 1$ $m g / l$, $\beta$-lactamase negative) and plasmid-mediated resistance (PPNG, MIC $\geqslant 1 \mathrm{mg} / \mathrm{l}$, $\beta$-lactamase positive).
(MIC $2 \mathrm{mg} / \mathrm{l}$ ). All isolates were very sensitive to cefotaxime (MIC $\leqslant 0.12 \mathrm{mg} / \mathrm{l})$, cefixime $(\mathrm{MIC} \leqslant 0 \cdot 12 \mathrm{mg} / \mathrm{l})$, spectinomycin $(\mathrm{MIC} \leqslant$ $32 \mathrm{mg} / \mathrm{l}$ ) and azithromycin (MIC $\leqslant 0.25$ $\mathrm{mg} / \mathrm{l})$. MIC distributions for these antibiotics are shown in table 1 .

Analysis of plasmids from the 16 PPNG revealed that two contained the $2.9 \mathrm{MDa}$ "Rio" plasmid, nine contained the $3.2 \mathrm{MDa}$ "African" plasmid and five contained the 4.4 MDa "Asian" plasmid. Table 2 lists relevant details of the patients from whom these isolates were obtained. The three PPNG isolates exhibiting high-level tetracycline resistance contained a $25 \cdot 2 \mathrm{MDa}$ plasmid, in addition to the $3.2 \mathrm{MDa}$ "African" $\beta$-lactamase plasmid, and gave a PCR product of the expected size when tested with primers to tet $M$.

Heterosexual male gonorrhoea accounted for 71 episodes (63\%), homosexual male gonorrhoea for four episodes $(3.5 \%)$ and female gonorrhoea for 38 episodes (33.5\%). One patient (female) admitted to being a sexworker. The male modal age was 29 years compared to a female modal age of 20 years. Postcode analysis demonstrated $90 \%$ of patients lived in Newham or surrounding East London boroughs. Ethnicity data are shown in table 3. Most patients (109) acquired their gonorrhoea in the UK but single cases of gonorrhoea were acquired in each of the following countries: Thailand, Ghana, Dominica and Nigeria. Male patients admitted to having more than one sexual partner in the previous three months in $54 \%$ of cases compared with $24 \%$ of women. A past history of gonorrhoea was obtained more often in men than in women $(39 \%$ vs. $18 \%)$ although the frequency of other past sexually transmitted diseases (STDs) was similar in both sexes (males $24 \%$, females $21 \%$ ). $C$ trachomatis and $T$ vaginalis were detected in $31 \%$ and $13.5 \%$ of females respectively. Only $16 \%$ of males had $C$ trachomatis cultured from their urethra. One female and one male patient had positive treponemal serology (both VDRL negative).

Most patients (103) received a clinic-supervised single oral dose of ciprofloxacin (250 $\mathrm{mg}$ ) at diagnosis. Three females were pregnant and received either Triplopen i.m. (1), which comprises a mixture of benzylpenicillin procaine penicillin and benethamine penicillin, or oral amoxycillin (2). Other antimicrobials used included spectinomycin i.m., doxycycline for presumed non-specific urethritis (3) and erythromycin. ${ }^{1}$ Two females failed to return for treatment of gonorrhoea diagnosed after routine culture, despite follow-up by a health advisor. Rates of return for follow-up tests of cure were satisfactory $(63 \%$ females, $71 \%$ males). Six of the 77 patients who returned showed evidence of persistent gonococcal infection using culture techniques. Within this group, two of the four females and both males were reinfected by untreated partners and had isolates fully sensitive to ciprofloxacin with which they were treated. The other two females were probably treatment failures as the gonococci isolated at follow-up had identical antibiograms to the 
Table 2 Patient epidemiological data in relation to plasmid profiles for the 16 PPNG isolates

\begin{tabular}{|c|c|c|c|c|c|c|}
\hline Sex & $\begin{array}{l}\text { Country of } \\
\text { birth }\end{array}$ & Ethnic group & Country acquired & $\begin{array}{l}\text { R-Lactamase } \\
\text { plasmid }(M D a)\end{array}$ & $\begin{array}{l}\text { TRNG plasmids } \\
(25 \cdot 2 \mathrm{MDa})\end{array}$ & $\begin{array}{l}\text { Conjugative } \\
\text { plasmid } \\
(24 \cdot 5 \mathrm{MDa})\end{array}$ \\
\hline$M$ & UK & Black-Caribbean & Dominica & $2 \cdot 9$ & No & Yes \\
\hline$M$ & UK & Black-African & UK & $3 \cdot 2$ & No & Yes \\
\hline$M$ & Ghana & Black-African & UK & $3 \cdot 2$ & No & No \\
\hline $\mathrm{F}$ & Ghana & Black-African & Ghana & $3 \cdot 2$ & No & No \\
\hline $\mathrm{F}$ & UK & White & UK (sex worker) & $3 \cdot 2$ & Yes & No \\
\hline$M$ & Nigeria & Black-African & Nigeria & $4 \cdot 4$ & No & Yes \\
\hline $\mathrm{F}$ & UK & Black-Caribbean & UK & $3 \cdot 2$ & Yes & No \\
\hline$M$ & Zimbabwe & Black-African & UK & $3 \cdot 2$ & No & Yes \\
\hline $\mathrm{F}$ & Zimbabwe & Black-African & UK & $3 \cdot \overline{2}$ & No & Yes \\
\hline$M$ & UK & Black-Caribbean & UK & $3 \cdot 2$ & Yes & No \\
\hline$M$ & Nigeria & Black-African & UK & $3 \cdot 2$ & No & Yes \\
\hline $\mathrm{F}$ & Barbados & Black-Caribbean & UK & $2 \cdot 9$ & No & Yes \\
\hline$M$ & Guyana & Black-Caribbean & UK & $4 \cdot 4$ & No & No \\
\hline $\mathrm{F}$ & Nigeria & Black-African & UK & $4 \cdot 4$ & No & No \\
\hline$M$ & Nigeria & Black-African & UK & $4 \cdot 4$ & No & No \\
\hline$M$ & Nigeria & Black-African & UK & $4 \cdot 4$ & No & No \\
\hline
\end{tabular}

All PPNG isolates possessed the $2 \cdot 6 \mathrm{MDa}$ cryptic plasmid.

Table 3 Ethnic groups of male and female patients with gonorrhoea

\begin{tabular}{lcc}
\hline $\begin{array}{l}\text { Ethnic } \\
\text { group }\end{array}$ & $\begin{array}{l}\text { Male } \\
(n=75)\end{array}$ & $\begin{array}{l}\text { Female } \\
(n=38)\end{array}$ \\
\hline White & $22(29 \%)$ & $23(60 \%)$ \\
Black-Caribbean & $41(55 \%)$ & $8(22 \%)$ \\
Black-African & $12(16 \%)$ & $3(8 \%)$ \\
Other & $0(0 \%)$ & $4(10 \%)$ \\
\hline
\end{tabular}

initial isolate in each case. The pre- and posttreatment isolates from each patient were subsequently shown to belong to the same auxotype-serovar class (data not presented). One female patient had pelvic inflammatory disease and received oral erythromycin, the other received Triplopen i.m. but her initial and subsequent isolates were found to be PPNG on laboratory testing.

\section{Discussion}

Analysis of patient postcodes confirmed that the GUM clinic served a very local patient population in which white $(40 \%)$ and blackCaribbean (43\%) ethnic groups were similarly represented. However, there was a marked difference observed when ethnicity was divided by sex (table 3 ). The reasons underlying this observation are not clear. Foreign travel was not an important factor in the acquisition of gonorrhoea, as most patients (109) had acquired infection in the UK. However, foreign travel and a high level of immigration may affect the antimicrobial resistance pattern of the strains circulating in the local gonococcal pool. This may explain the finding of a higher proportion of PPNG $(15 \%)$ amongst the isolates compared with that reported from other London GUM clinics: $4 \%$ at the Royal London Hospital, Whitechapel ${ }^{5}$ and $5 \%$ at St. Mary's Hospital, Paddington $^{6}$. Three of the four isolates acquired abroad were PPNG, the other a CMRNG (penicillin MIC $=2 \mathrm{mg} / \mathrm{l}$ ). All of these "foreign" isolates were sensitive to ciprofloxacin, spectinomycin, azithromycin, cefixime and cefotaxime.

The ratio of male:female patients was $2: 1$, which is similar to that seen on KC60 returns for total English gonorrhoea cases ${ }^{7}$. The asymptomatic nature of gonorrhoea in some women may account for non-attendance at GUM clinics. Homosexually-acquired gonorrhoea accounted for only four (3.5\%) of the total cases seen. All these were from white patients and were isolated from the urethra only; pharyngeal and rectal sites failing to grow the organism. All four admitted unprotected oral and anal intercourse within the past three months. Recent concern has been expressed over the increase in homosexual male gonorrhoea case numbers. ${ }^{8}$ Homosexually-acquired gonorrhoea accounts for $12 \%$ of total gonorrhoea cases at a nearby teaching hospital (unpublished observations). The reasons underlying the small number of homosexual men seen at this clinic probably reflects the strong representation of blackCaribbean and black-African ethnic groups in the local population amongst whom homosexual practice is either less frequent or not openly acknowledged. In addition, there is a tendency for homosexual men to attend the larger GUM clinics attached to London teaching hospitals.

This study supports others suggesting that a past history of gonorrhoea and other STDs are risk factors for re-acquisition of gonorrhoea. ${ }^{9}$ The rates of $C$ trachomatis isolation in heterosexual males and females with gonorrhoea compare favourably with previous studies. ${ }^{10} T$ vaginalis was demonstrated in $13.5 \%$ of female patients. The two patients with positive treponemal serology were born overseas; a Jamaican male (30 years) and a Ghanaian female. ${ }^{29}$ Neither had a history of syphilis and both were treated appropriately with i.m. Triplopen.

Until recent years the standard first-line therapy for gonococcal infection in London GUM clinics consisted of single high dose oral amoxycillin with probenecid. The treatment of gonorrhoea has since become more difficult owing to the increased prevalence of CMRNG and PPNG. Today, fluoroquinolones such as ciprofloxacin have become popular first-line agents and shown to be suitable replacement for the high dose amoxycillin plus probenecid regimen. Unfortunately, there have been recent reports of ciprofloxacin resistant gonococci with associated clinical failure in London. ${ }^{11}$ Currently, fewer than $1 \%$ of clinic isolates exhibit 
decreased susceptibility to ciprofloxacin, ${ }^{12}$ and some of these may still respond to a high dose therapy. Only one study isolate exhibited decreased susceptibility (MIC $=0.06 \mathrm{mg} / \mathrm{l}$ ) but the patient was cured microbiologically and clinically at both one and three weeks after ciprofloxacin therapy. Other alternative treatments for gonorrhoea include spectinomycin and broad-spectrum cephalosporins (such as cefotaxime or cefixime). Spectinomycin resistance is now very rare in the UK, but occurs more commonly in South East Asia. ${ }^{13}$ Resistance to broad-spectrum cephalosporins is rare worldwide. Azithromycin is a new macrolide with combined activity against both $N$ gonorrhoeae and $C$ trachomatis and has the advantage of single dose oral therapy for both infections. Although tetracyclines are not used as a first-line therapy for gonorrhoea in the UK, they are frequently used to treat nonspecific urethritis. Since 1985, there has been a marked increase in the number of gonococci exhibiting resistance to tetracycline due to acquisition of a $25 \cdot 2 \mathrm{MDa}$ plasmid and early reports from Holland ${ }^{14}$ showed an association with $\beta$-lactamase $3 \cdot 2 \mathrm{MDa}$ plasmid carriage, as was found in this study.

Ciprofloxacin replaced high dose oral amoxycillin and probenecid as first-line therapy at Newham General Hospital in 1987 following an observed increase in treatment failures with the amoxycillin regimen. The susceptibility data support continued use of ciprofloxacin as first-line clinic therapy. The current high PPNG rate implies an unacceptable treatment failure rate if the traditional amoxycillin plus probenecid regimen were re-introduced as first-line therapy. Spectinomycin, cefixime, cefotaxime and azithromycin all appear effective alternative therapies. This work emphasises the need for continued sur- veillance of antibiotic susceptibility patterns of $N$ gonorrhoeae if a successful therapeutic outcome is to be achieved.

We are grateful to the staff of both the Genitourinary Medicine Clinic and the Microbiology Department of Newham General Hospital for their support. Antibiotics for laboratory testing were kindly provided by Lederle Laboratories (cefixime), Bayer U.K. Ltd. (ciprofloxacin) and Pfizer Ltd. (azithromycin).

1 STD treatment strategies. WHO consultation on development of sexually transmitted disease treatment strategies. 1989 WHO/VDT/89.477.

2 Centers for Disease Control. Tetracycline-resistant Neisseria gonorrhoeae-Georgia, Pennsylvania, New Neisseria gonorrhoeae-Georgia, Pentire. MMWR 1985;34:563-70.

3 Birnboim HC, Doly J. A rapid alkaline extraction procedure for screening recombinant plasmid DNA. Nucleic Acid Res 1979;7:1513-22.

4 Ison CA, Tekki N, Gill MJ. Detection of the tetM determinant in Neisseria gonorrhoeae. Sex Trans Dis 1993;20 329-33.

5 Lewis DA, Forster GE, Hooi AY, Goh BT, Wisdom AR. Gonococcal infection in east London: A two centre microbiological and clinical study. Poster abstract No 85 at the M.S.S.V.D. Spring Meeting, 12-15 May 1994 Liverpool, UK.

6 Ison CA, Easmon CSF. Changes in penicillinase-producing Neisseria gonorrhoeae isolated in London. $7 \mathrm{Med}$ Microbiol 1989;30:239-44.

7 Department of Health. New cases seen at NHS genitourinary medicine clinics in England: 1991 annual figures.

8 Riley VC. Resurgent gonorrhoea in homosexual men. Lancet 1991;337:183.

9 Lundin RS, Wright MW, Scatliff JN. Behavioural and social characteristics of the patient with repeated venereal disease and his effect on the statistics of venereal disease. $B r$ F Venereal Dis 1977;53:140-4.

10 Batteiger BE, Jones RB. Chlamydial infections. Infect Dis Clin North Am 1987;1:55-81.

11 Gransden WF, Warren CA, Phillips I, Hodges M, Barlow D. Decreased susceptibility of Neisseria gonorrhoeae to D. Decreased susceptibility of
ciprofloxacin. Lancet 1990;1:51.

12 Ison CA, Branley NS, Kirtland K, Easmon CSF. Ison CA, Branley NS, Kirtland $\mathrm{K}$, Easmon CSF. Neisseria gonorrhoeae. BMF 1991;303:1307.

13 Clendennen TE, Echeverria P, Saengeur S, Kees ES Boslego JW, Wignall FS. Antibiotic susceptibility survey of Neisseria gonorrhoeae in Thailand. Antimicrob Agents Chemother 1992;36:1682-7.

14 Roberts MC, Wagenvoort JHT, van Klingeren B, Knapp JS. TetM- and $\beta$-lactamase-containing Neisseria gonorrhoeae (tetracycline resistant and penicillinase producing) in the Netherlands. Antimicrob Agents Chemother 1988;32:158. 